\title{
Prevalência e severidade da doença periodontal e acúmulo de biofilme dentário em crianças da Clínica de Odontopediatria da UFPB
}

\author{
Prevalence and severity of the periodontal disease and accumulation \\ dental biofilm in children from the Paediatric Dentistry Clinic at UFPB
}

\author{
* Rossana Vanessa Dantas de Almeida \\ * Érika de Morais Beltrão \\ * Carolina Bezerra Cavalcanti Nóbrega \\ ** Ana Maria Gondim Valença
}

\begin{abstract}
RESUMO
Este trabalho objetivou verificar a prevalência e a severidade da doença periodontal em pacientes da Clínica de Odontopediatria da Universidade Federal da Paraíba, relacionando os níveis de doença com o gênero e o acúmulo de biofilme. Compôs a amostra 64 crianças na faixa etária de 09 a 11 anos que estavam em atendimento na referida Clínica. Foram coletados, por uma única examinadora, índices de biofilme dentário (IHOS e O'leary) e doença gengival (ISG), e registradas a presença de recessão gengival e de bolsa periodontal. Os dados foram analisados com o auxílio do programa SPSS e tratados estatiscamente pelos testes do Qui-quadrado e Exato de Fisher $(p<0,05)$. Os resultados mostram as prevalências: gengivite $100 \%$; recessão gengival $21,8 \%$; bolsa periodontal 4,6\%. Níveis de higiene oral insatisfatórios foram encontrados em $89,1 \%$ e $54,7 \%$ das crianças pelos índices de O’leary e IHOS, respectivamente. A presença de gengivite, recessão gengival e bolsa periodontal mostraram-se respectivamente em $62,2 \%, 16,2 \%$ e $5,4 \%$ no gênero feminino e $59,3 \%, 29,6 \%$ e $3,7 \%$ no gênero masculino, não observando diferença estatisticamente significante de acometimento das alterações entre os gêneros. 0 acúmulo de biofilme mostrou níveis insatisfatórios pelos índices de O'leary e IHOS, respectivamente, em 88\% e 54,1\% para o gênero feminino e 89\% e 55,6\% para o masculino, não observando diferença estatística entre os gêneros. Da mesma forma, não foi possível observar relação entre o acúmulo de biofilme dentário e a presença de gengivite severa. A partir dos resultados concluí-se que: as condições de higiene oral foram insatisfatórias; a presença e severidade da gengivite se mostraram elevadas, bem como as prevalências de recessão gengival e de bolsa periodontal; não houve predileção das alterações segundo o gênero e não pode ser observada associação estatisticamente significante entre o acúmulo de biofilme e os padrões de gengivite.
\end{abstract}

Palavras-chave:

Higiene bucal, Doenças da gengiva, Periodontite

\section{INTRODUÇÃO}

Assim como a cárie dentária, a doença periodontal também é uma das patologias mais freqüentes nos seres humanos, sendo esta uma alteração de grande importância epidemiológica para a Odontologia, atingindo todas as faixas etárias. Apesar disso, foi por muito tempo negligenciada, principalmente em crianças e adolescentes. Entretanto, com o declínio da prevalência da cárie, a preocupação com os problemas periodontais na população infantil aumentou (CARDOSO; ROSING; KRAMER, 2000).

É sabido que a prevalência de gengivite é bastante elevada em pacientes infantis e, quando esta não é diagnosticada e eliminada durante a infância, permanece e acompanha o desenvolvimento do paciente até a fase adulta, podendo levar ao estabelecimento de um quadro mais sério, a periodontite (JAHN; JAHN, 1997; MORAES, 2001).

A doença periodontal em crianças e adolescentes mostra pouco ou mesmo a ausência de sintomas subjetivos, acarretando gran- de risco de negligência nos estágios iniciais (KOCH et al., 1992). Outra alteração periodontal, também diagnosticada na infância, é a recessão gengival que pode ser considerada uma expressão clínica da perda de inserção do periodonto, permitindo sua utilização como um diagnóstico auxiliar da susceptibilidade à doença periodontal $(\mathrm{BECH}$; KOCH, 1994).

A gengivite, bem como a periodontite são doenças infecciosas causadas por bactérias que colonizam a superfície dos dentes, formando o biofilme dentário. A relação existente entre o acúmulo de biofilme dentário e a inflamação gengival está bem estabelecida desde os estudos de Löe (BUISCHI; AXELSSON, 1997).

Dessa forma, o presente trabalho objetivou verificar a prevalência e a severidade da doença periodontal em pacientes da Clínica de Odontopediatria da Universidade Federal da Paraíba, relacionando os níveis de doença com o gênero e o acúmulo de biofilme dentário.

\section{REVISÃO DA LITERATURA}

Vários estudos mostram a susceptibilidade da criança à doença periodontal, principalmente às gengivites, que tem uma média de prevalência variando entre $40 \%$ a $100 \%$ (COUTINHO; TOSTES, 1997; SARIAN; DUARTE; CARVALHO, 1997; SUSIN, AZEVEDO, RÖSING, 1997; CUNHA; CHAMBRONE, 1998; SILVA; RÖSING, 1999; BARROSO; TOLEDO; SOUZA, 2000; CARDOSO, RÖSING, KRAMER, 2000; COSTA et al., 2000; CHARLIER; BARCELOS; VIANNA, 2002).

Observando a prevalência de doença gengival em crianças da Clinica de Odontopediatria da Universidade Federal Fluminense (Niterói/RJ) pelo índice de sangramento gengival mensurado pelos acadêmicos do $6^{\circ}$ período da graduação, Coutinho e Tostes (1997) verificaram a prevalência de doença gengival em crianças na faixa etária de 4 a 12 anos utilizando o índice de sangramento gengival. Os resultados encontrados mostraram presença de 
gengivite em $83,3 \%$ da amostra estudada e, $88,1 \%$ na faixa etária de 9 a 11 anos. As mesmas autoras avaliando ainda a severidade da doença gengival na mesma faixa etária, identificaram $69,2 \%$ das crianças com gengivite leve, ou seja, de 01 a 15 superfícies gengivais apresentando sangramento. No que se refere à distribuição da doença gengival pelo gênero, verificou-se haver discretas diferenças percentuais entre os mesmos no que diz respeito aos distintos graus de gengivite, de forma que a gengivite suave foi mais prevalente no gênero feminino que no masculino; o oposto ocorreu com relação aos quadros moderado e severo, uma vez que nestas modalidades houve maior prevalência do gênero feminino.

Cunha e Chambrone (1998), estabelecendo a prevalência e severidade da gengivite pelo Índice Gengival em crianças de 7 a 14 anos de idade com baixo nível sócio econômico, verificaram uma freqüência de $99,2 \%$ na faixa etária de 9 a 11 anos. A severidade da doença foi considerada compatível com uma inflamação moderada, encontrando em faixas etárias precoces uma alta prevalência de gengivite com baixa severidade e não havendo diferença estatisticamente significante entre os gêneros, apesar das crianças do gênero masculino apresentarem maior frequiência em intervalos de severidade leve enquanto que as do gênero feminino predominaram em intervalos de gravidade pouco maior.

Costa et al. (2000) identificaram a presença da doença periodontal através da conjugação de parâmetros clínicos (sangramento à sondagem, alterações da morfologia gengival, presença de cálculo e profundidade de sondagem) e radiográficos (perda óssea) entre 360 crianças de uma escola pública de Belo Horizonte (MG). Dessa forma, observaram uma freqüência de gengivite em $98,9 \%$ das crianças (6 a 10 anos) e $100 \%$ dos adolescentes (11 a 15 anos) e cálculo dentário em 18,9\% das crianças e 58,9\% dos adolescentes, indicando um baixo nível de higiene oral destes escolares.

No estudo de Cardoso, Rösing e Kramer (2000), realizado em Pareci Novo (RS), com crianças entre 6 e 12 anos foi diagnosticada gengivite em $100 \%$ dos casos e esta alteração periodontal apresentou correlação positiva com a presença de biofilme dentário. No mesmo trabalho os autores verificaram a presença de biofilme visível em 100\% das crianças examinadas, indicando a necessidade de medidas coletivas de promoção de saúde que envolvam a instrução para o controle da biofilme dentário nesta população.

Maltz e Silva (2001), buscando determinar a relação entre o nível sócio-econômico e a prevalência de gengivite por meio do índice de sangramento gengival, examinaram mil escolares de 12 anos de idade provenientes de escolas das redes de ensino pública e particular verificando valores do referido índice de $14,7 \%$ e $21,7 \%$ para os escolares das redes particular e pública respectivamente. Porém não foi possível observar correlação entre a presença de gengivite e o nível sócio-econômico.

Identificando a presença de recessão gengival em 201 escolares na faixa etária de 9 a 12 anos matriculados em escolas da rede pública da cidade de Porto Alegre (RS), Susin, Azevedo e Rösing (1997) observaram um acometimento da alteração periodontal em 8,9\% da amostra estudada, sendo ela encontrada em 7,5\% dos escolares de 9 a 11 anos. Os autores concluíram que pacientes portadores de recessão gengival nesta idade devem ser observados de forma minuciosa e frequiente por constituírem um grupo de risco a problemas periodontais.

Silva e Rösing (1999), em seu trabalho, objetivaram verificar a prevalência de perda de inserção periodontal em adolescentes de 12 a 16 anos de idade de Porto Alegre (RS) por meio de sondagem periodontal nos ângulos mésio-vestibulares, disto-vestibulares e no centro das faces vestibulares e linguais/ palatinas dos incisivos e primeiros molares permanentes. Assim, os autores identificaram uma frequiência de perda de inserção periodontal na amostra de $71,8 \%$, sendo $55,6 \%$ em escolares de 12 anos de idade.

No estudo de Costa et al. (2000) foi verificada a profundidade de sondagem gengival de até $3,5 \mathrm{~mm}$ em crianças (6 a 10 anos) e adolescentes (11 a 15 anos), observando uma freqüência de $71,6 \%$ e $57,2 \%$, respectivamente. Os autores observaram ainda a presença de perda óssea por meio de radiografias padronizadas realizadas pela técnica do paralelismo, em 2,2\% entre crianças e $6,1 \%$ entre adolescentes revelando, portanto, que a destruição periodontal pode ocorrer em qualquer idade na dentição decídua, mista e/ou permanente.

Dessa forma, o profissional de Odontologia deve ficar atento para o diagnóstico precoce da doença periodontal, em especial a gengivite, que na infância pode ser encontrada e evoluir atingindo a idade adulta.

\section{MATERIAIS E MÉTODOS}

O presente estudo foi aprovado pelo Comitê de Ética em Pesquisa do Centro de Ciências da Saúde da Universidade Federal da Paraíba.

Utilizou-se uma amostra de 64 crianças na faixa etária de 09 a 11 anos que estavam em atendimento clínico durante os semestres do ano letivo 2002. Não foram incluídos na amostra pacientes portadores de acessórios ortodônticos fixos e/ou removíveis e que estavam fazendo uso de medicamentos anticonvulsivante ou possuíssem doença sistêmica.

Realizou-se exame clínico nas dependências da Clínica de Odontopediatria durante os dias de atendimento da criança por uma única examinadora previamente treinada (mediante exame clínico de pacientes na mesma faixa etária da avaliada no presente estudo) sobre cada índice, tendo a colaboração de uma anotadora.

Foram mensuradas as seguintes condições: 1. acúmulo de biofilme dentário -pelos índices de Higiene Oral Simplificado (GREENE; VERMILLION, 1964) e O'Leary (O'LEARY; DRAKE; NAYLOR, 1972); 2. doença gengival - por meio do Índice de Sangramento Gengival (AINAMO; BAY, 1975); 3. recessão gengival; 4. bolsa periodontal.

Dentre os índices coletados, o Índice de Higiene Oral Simplificado (IHOS) mede a existência de biofilme dentário previamente corado em superfícies de seis elementos dentários pré-estabelecidos. O Quadro 1 apresenta os escores obtidos para o IHOS e sua interpretação.

Outro indicador empregado para mensurar a quantidade de biofilme dentário foi o Índice de O'Leary, obtendo-se uma segunda avaliação clínica das condições de higiene oral das crianças. Após a revelação, foram registradas as superfícies dentárias coradas, calculando-se o percentual de superfícies recobertas. Considerou-se com condição de higiene oral satisfatória aquelas que obtiveram percentual de superfícies coradas igual ou inferior a 30\% (Quadro 1).

A doença gengival foi mensurada pelo Índice de Sangramento Gengival (ISG) o qual baseia-se no critério simples: se a gengiva sangra ou não sob sondagem suave. Nesta avaliação utilizou-se a sonda periodontal CPI/ WHO e as orientações descritas no manual do examinador do Projeto SB2000 (BRASIL, 2000). Desta forma, os elementos dentários foram submetidos à sondagem nas suas superfícies vestibular e lingual (palatina), iniciando-se pela região distal até alcançar a área mesial. A sonda foi introduzida levemente no sulco gengival (ou na bolsa periodontal), com ligeira inclinação em relação ao longo eixo do dente, sendo executados movimentos de vai-e-vem de pequena amplitude. $\mathrm{O}$ índice foi analisado segundo a classificação de Coutinho e Tostes (1997) que consideram a quantidade de superfícies gengivais sangrantes (Quadro 1).

A recessão gengival foi avaliada pelo do método visual, onde se considerou como normalidade a margem gengival localizada em uma posição coronal à junção amelocementária (WATSON, 1984).

A presença de bolsa periodontal foi verificada pela mẹsuração da profundida- 
de do sulco gengival com sonda periodontal (WHO/CPI), registrando a ocorrência desta diante de valores iguais ou superiores a 3 mm (KOPCZYK; LENOX, 1973). Uma vez que a sonda WHO/CPI tem uma esfera de $0,5 \mathrm{~mm}$ na sua extremidade e área anelada em preto situada entre 3,5 a $5,5 \mathrm{~mm}$ da ponta, considerou-se a presença de bolsa periodontal quando a margem gengival dos sítios se localizava nesta área demarcada na cor preta. Estes elementos dentários eram então submetidos a radiografias interproximais para verificação e quantificação da perda óssea.

Os resultados obtidos foram analisados estatisticamente com o auxilio do software SPSS versão 9.0, utilizando-se os testes do Qui-quadrado e Exato de Fisher, com nível de significância de 5\%.

\section{RESULTADOS}

Foram examinadas 64 crianças, sendo 37 do gênero feminino e 27 do masculino. As freqüências de crianças examinadas conforme a severidade da doença gengival são vistas na tabela 1 .

Procurando relacionar as alterações (gengivite, recessão gengival e bolsa periodontal) que acometem o periodonto infantil conforme o gênero é apresentada a tabela 2 .

A tabela 3 mostra a distribuição das condições de acúmulo de biofilme dentário mensuradas pelos índices de O'Leary e IHOS conforme o gênero.

Os padrões de gengivite foram relacionados com os índices de biofilme de forma a identificar possíveis relações existentes entre o acúmulo de biofilme dentário e a inflamação gengival (Tabela 4).

\section{DISCUSSÃO}

Vários estudos mostram os altos níveis da doença periodontal acometendo pacientes infantis, em especial a doença gengival (COUTINHO; TOSTES, 1997; CUNHA; CHAMBRONE, 1998; COSTA et al., 2000; CARDOSO; RÖSING; KRAMER, 2000). Este fato foi também evidenciado no presente estudo, onde se observou $100 \%$ de gengivite na amostra estudada, coincidindo com os achados de Cardoso, Rösing e Kramer (2000). Da mesma forma, a alteração se mostrou mais freqüente na condição grave a gengivite severa com 60,9\% (Tabela 1).

Para a análise estatística foi necessário agrupar condições de gengivite leve e moderada como regulares, uma vez que não houve, na amostra estudada, crianças com ausência de doença gengival. A comparação entre os gêneros em função do acometi-
Quadro 1: Critérios de interpretação dos escores obtidos pelos Índices de O'Leary, IHOS e ISG.

\begin{tabular}{lccc} 
Índices & Escores & Condição & Interpretação \\
IHOS & 0 a 1 & Higiene Oral Boa & Satisfatória \\
& 1,1 a 3,0 & Higiene Oral Deficiente & Insatisfatória \\
\hline O'Leary & 0 a $30 \%$ & Higiene Oral Boa & Satisfatória \\
& $>30 \%$ & Higiene Oral Deficiente & Insatisfatória \\
\hline ISG & 0 & Gengivite Ausente/ & Satisfatória \\
& $><35$ & Gengivite Leve/Moderada & Regular \\
& $>35$ & Gengivite Severa & Insatisfatória
\end{tabular}

Tabela 1: Distribuição das crianças segundo a severidade da doença gengival. João Pessoa, 2002

\begin{tabular}{|l|cc|}
\hline \multirow{2}{*}{ Condição Gengival } & \multicolumn{2}{|c}{ Crianças examinadas } \\
\cline { 2 - 3 } & $\mathbf{n}$ & $\%$ \\
\hline Gengivite ausente & 0 & 0 \\
Gengivite leve & 01 & 1,6 \\
Gengivite moderada & 24 & 37,5 \\
Gengivite severa & 39 & 60,9 \\
\hline Total & $\mathbf{6 4}$ & $\mathbf{1 0 0}$ \\
\hline
\end{tabular}

Tabela 2: Distribuição das crianças segundo o acometimento de doença gengival, recessão gengival e bolsa periodontal pelo gênero. João Pessoa, 2002

\begin{tabular}{l|c|c|c|c|c|r}
\hline Condição & \multicolumn{2}{|c|}{ Feminino } & \multicolumn{2}{c|}{ Masculino } & \multicolumn{2}{c}{ Total } \\
\cline { 2 - 7 } & n & \% & n & \% & n & \% \\
\hline Gengivite ausente/ & & & & & & \\
leve/ moderada & 14 & 37,8 & 11 & 40,7 & 25 & 39,1 \\
Gengivite severa & 23 & 62,2 & 16 & 59,3 & 39 & 60,9 \\
Recessão ausente & 31 & 83,8 & 19 & 70,4 & 50 & 78,2 \\
Recessão presente & 6 & 16,2 & 8 & 29,6 & 14 & 21,8 \\
Ausência de bolsa & & & & & & \\
periodontal & 35 & 94,6 & 26 & 96,3 & 61 & 95,4 \\
Presença de bolsa & & & & & & \\
periodontal & 2 & 5,4 & 1 & 3,7 & 03 & 4,6
\end{tabular}

Testes do Qui-quadrado e Exato de Fisher - $p>0,05$

Tabela 3: Distribuição das crianças segundo o acúmulo de biofilme pelos índices de O'Learye IHOS pelo gênero. João Pessoa, 2002

\begin{tabular}{ll|c|c|c|c|c|c}
\hline \multirow{2}{*}{ Higiene Oral } & \multicolumn{2}{|c|}{ Feminino } & \multicolumn{2}{c|}{ Masculino } & \multicolumn{2}{c}{ Total } \\
\cline { 2 - 7 } & n & \% & n & \% & n & \% \\
\hline \multirow{2}{*}{ O’leary } & Satisfatória & 2 & 5,4 & 5 & 07 & 07 & 10,9 \\
& Insatisfatória & 35 & 94,6 & 22 & 57 & 57 & 89,1 \\
\hline \multirow{2}{*}{ IHOS Satisfatória } & 17 & 45,9 & 12 & 29 & 29 & 45,3 \\
& Insatisfatória & 20 & 54,1 & 15 & 35 & 35 & 54,7
\end{tabular}

Testes do Qui-quadrado e Exato de Fisher - $p>0,05$

Tabela 4: Distribuição das crianças segundo o acúmulo de biofilme mensurado pelos Índices de O'Leary e IHOS pelo padrão de gengivite. João Pessoa, 2002

\begin{tabular}{ll|c|c|c|c}
\hline \multirow{2}{*}{ Higiene Oral } & \multicolumn{2}{|c|}{ Leve/Moderada } & \multicolumn{2}{c}{ Severa } \\
\cline { 3 - 6 } & & $\mathbf{n}$ & $\%$ & $\mathbf{n}$ & $\%$ \\
\hline \multirow{2}{*}{ O’leary } & Satisfatória & 3 & 12,0 & 4 & 10,2 \\
& Insatisfatória & 22 & 88,0 & 35 & 89,8 \\
\hline \multirow{2}{*}{ IHOS } & Satisfatória & 11 & 44,0 & 18 & 46,1 \\
& Insatisfatória & 14 & 66,0 & 21 & 53,9 \\
\hline
\end{tabular}

Testes do Qui-quadrado e Exato de Fisher - $p>0,05$. 
mento da doença gengival mostrou a condição insatisfatória (gengivite severa) com maior prevalência no gênero feminino $(62,2 \%)$, apesar de não ter sido observada diferença estatisticamente significante (Tabela 2).

Foi identificada a presença de recessão gengival em $21,8 \%$ das crianças, sendo esta mais prevalente no gênero masculino $(29,6 \%)$ do que no feminino $(16,2 \%)$, porém não observando diferença estatística (Tabela 2). Esta prevalência se mostrou superior àquela relatada por Susin, Azevedo e Rösing (1997) - 7,5\%. Os resultados do presente estudo demonstram o grupo de risco à doença periodontal que estas crianças fazem parte.

A presença de bolsa periodontal foi observada em 4,6\% das crianças (Tabela 2), não havendo diferença estatística na ocorrência desta alteração entre os gêneros. $\mathrm{O}$ resultado obtido é semelhante aquele relatado por Costa et al. (2000).

Os níveis de higiene oral se mostraram divergentes entre os dois índices (O'Leary e IHOS), porém ambos revelaram expressivo acúmulo de biofilme, fato que denota uma condição de higiene oral insatisfatória nas crianças avaliadas (Tabela 3). Em relação a este aspecto, não foi observada diferença estatisticamente significante para os gêneros masculino e feminino.

Não foi encontrada relação entre os padrões de gengivite e os índices de biofilme, ou seja, o seu acúmulo ocorreu tanto naquelas crianças que mostraram padrões de gengivite moderada/leve quanto severa (Tabela 4).

\section{CONCLUSÕES}

De acordo com a metodologia empregada e os resultados obtidos no presente estudo, é possível concluir:

1. As condições de higiene oral das crianças avaliadas foram insatisfatórias;

2. A prevalência e a severidade da gengivite se mostraram elevadas na amostra estudada, bem como a presença de recessão gengival e de bolsa periodontal;

3. 0 acúmulo de biofilme dentário e presenças de sangramento gengival, recessão gengival e bolsa periodontal não foram influenciados pelo gênero das crianças;

4. Não foi observada associação entre o acúmulo de biofilme e os padrões de gengivite.

\section{ABSTRACT}

The aim of this work was to verify the prevalence and severity of periodontal disease in patients between 9 and 11 years old from the Paediatric Dentistry Clinic of the Federal University of Paraiba, relating the levels of disease with gender and biofilm accumulation. The sample was composed by 64 children that were in attendance on this
Clinic. One examiner carried out the exam. There was dental biofilm collect indexes (OHI-S and O'leary), bleeding index (ISG), and it was registered the presence of gingival recession and pocket depth. The data were submitted to a statistical analysis with the aid of the SPSS program and treated by the tests of the Qui-square and Exact of Fisher ( $p$ $<0,05)$. The results show the following prevalence: gingivitis $100 \%$; recession gingival $21.8 \%$; pocket depth $4.6 \%$. It was observed unsatisfactory oral hygiene level in $89.1 \%$ and $54.7 \%$ of the children by the O'leary and OHI-S indexes, respectively. The presence of gingivitis, gingival recession and pocket depth were shown respectively in $62.2 \%, 16.2 \%$ and $5.4 \%$ in the feminine gender and $59.3 \%, 29.6 \%$ and $3.7 \%$ in the masculine gender, not observing statistical difference of the alterations among the gender. The biofilm accumulation showed unsatisfactory level by O'leary and OHI-S indexes, respectively, in $88 \%$ and $54,1 \%$ for the female gender and $89 \%$ and $55,6 \%$ for the mate, do not observing differences between the genders. In the same way, it was not possible to observe relationship between the accumulation of dental biofilm and the presence of severe gingivitis. The conclusion was that: the conditions of oral hygiene were unsatisfactory; the presence and severity of the gingivitis were shown high, as well the prevalence of gingival recession and pocket depth; there was not predilection of the alterations according to the gender and significant association could not be observed between the biofilm accumulation and the gingivitis levels.

\section{Keywords}

Periodontitis, Oral hygiene, Gingival diseases

\section{REFERÊNCIAS}

AINAMO, J.; BAY, I. Problems and Proposals for Recording Gingivitis and Plaque. Int. Dent. J., London, v. 25, no. 4, p. 229-35, Dec. 1975.

BARROSO, E. M.; TOLEDO, B. E. C.; SOUZA, T. H. R. Avaliação da Reabsorção Óssea Periodontal Através de Radiografias Interproximais em Escolares de 11 a 14 Anos. J. Bras. Odontoped. Odontol. Bebê, Curitiba, v. 3, n. 13, p. 215-218, maio/jun. 2000.

BECK, J. D.; KOCH, G. G. Characteristics of Older Adults Experiencing Periodontal Attachment Loss as Gingival Recession or Probing Depth. J. Periodontol. Res, Copenhagen, v. 29, no. 4, p. 290-298, July. 1994.
BRASIL, Ministério da Saúde. Secretaria de Políticas de Saúde. Departamento de Atenção Básica. Área Técnica em Saúde Bucal. Projeto SB2000: Condições de Saúde Bucal da População Brasileira no ano de 2000. Brasília, 2000. 56p. Disponível em: < http:// www.saude.gov.br/ programas/bucal/principal.htm $>$. Acesso em: 18 out. 2000.

BUISCHI, Y. P.; AXELSSON, P. Controle Mecânico da Placa Dental Realizado pelo Paciente. In: Kriger, L. Promoção da Saúde Bucal. São Paulo: Artes Médicas, 1997. P. 115-127.

CARDoSO, L.; ROSING, C. A.; KRAMER, T. F. Doença Periodontal em Crianças - Levantamento Epidemiológico Através dos Índices de Placa Visível e de Sangramento Gengival. J. Bras. Odontoped. Odontol. Bebê, Curitiba, v. 3 , n. 11, p. 55-61, jan./fev. 2000 .

CHARLIER, S. C.; BARCELOS, R.; VIANNA, R. B. C. Periodontite de Acometimento Precoce - Diagnóstico em Odontopediatria. J. Bras. Odontoped. Odontol. Bebê, Curitiba, v. 5, n. 23, p. 75-79, jan./fev. 2002

COSTA, F. O. et al. Prevalência de Doença Periodontal de Início Precoce em Crianças e Adolescentes de uma Escola Pública em Belo Horizonte (MG). Revista do CROMG, Belo Horizonte, v. 6, n. 1, p.53-62, jan./abr. 2000.

Coutinho, T. C.; Tostes, M. A. Prevalência de Gengivite em Crianças. Rev. Gaúcha Odontol., Porto Alegre, v. 45, n. 3, p. 170-174, maio/jun. 1997.

CUnHA, A. C. P.; CHAmbrone, L. A. Prevalência de Gengivite em Crianças de um Nível Social Baixo. Rev. Periodontia, São Paulo, v. 7, n. 1, p. 6-10, jan./abr. 1998.

GREENE, J. C.; VERMILLION, J. R. The Simplified Oral Hygiene Index. J. Am. Dent. Assoc., Chicago, v. 68, no.1, p. 7-13, Jan. 1964.

JAHN, M. R.; JANH, R. S. Fique Atento: Criança Também tem Gengivite. Rev. Assoc. Paul. Cirurg. Dent., São Paulo, v. 51, n. 4, p. 355-58, jul./ago. 1997.

KOCH, G. et al. Doença Periodontal. In: . Odontopediatria: Uma Abordagem Clínica. São Paulo: Santos, 1992. P. 211- 224. 
KOPCZYK, R. A.; LENOX, J. A. Periodontal Health and Disease in Children: Examination and Diagnosis. Dent. Clin. North Am., Philadelphia, v. 17, no. 1, p. 25-33, Jan. 1973.

MALTZ, M.; SILVA, B. B. Relação Entre Cárie, Gengivite e Fluorose e Nível Socioeconômico em Escolares. Rev. Saúde Pública, São Paulo, v. 35, n. 2, p. 170-76, abr. 2001.

MORAES, E. S. Prevalência de Gengivite e Periodontite em Crianças na Faixa Etária de 3 a 5 Anos na Cidade de Aracajú (SE). 2001. 119f. Dissertação (Mestrado em Odontologia) Universidade Federal da Paraíba, João Pessoa.

O'LEARY, T. J.; DRAKE, R. B.; NAYLOR, J. E. The Plaque Control Record. J. Periodontol., Chicago, v. 43, no.1, p. 38, Jan.1972.

SARIAN, R.; DUARTE, C. A.; CARVALHO, J. C. C. Doenças Periodontais na Infância e Adolescência. In: GUEDESPINTO, A. C. Odontopediatria. 6. ed. São Paulo: Santos, 1997. P. 323-353.

SILVA, C. M.; RÖSING, C. K. Prevalência de Perda de Inserção Periodontal em Adolescentes de 12 a 16 Anos em uma Escola Pública de Porto Alegre. Rev. Fac. Odontol., Porto Alegre, v. 40, n. 1, p. 29-32, set. 1999

SUSIN, C.; AZEVEDO, M. P.; ROSING, C. K. Prevalência de Recessão Gengival em Escolares e sua Relação com o Estado Inflamatório Gengival. Rev. Periodontia, São Paulo, v. 6, n. 1, p. 41-45, jan./jun. 1997.

WATSON, P. J. C. Gingival Recession. J Dent., Oxford, v.12, no.1, p.29-35, Mar.1984.

\section{Endereço para correspondência:}

Rossana Vanessa Dantas de Almeida

Rua Luiz Germóglio, 439/304 - Bancá-

rios

João Pessoa - PB - 58051-742

e-mail: rossanacd@hotmail.com 